\title{
Site-Specific Transition Metal 3d Electron Configurations Studied by Energy Loss with Channeled Electrons
}

\author{
K. Tatsumi, ${ }^{*}$ S. Muto, ${ }^{*}$ and J. Rusz,** \\ * Graduate School of Engineering, Nagoya University, Nagoya 464-8603, Japan \\ **Department of Physics and Astronomy, Uppsala University, Box 516, SE-75120 Uppsala, Sweden
}

Not by focusing the electron beam to atomic size, but by utilizing standing waves propagating through a set of specific atom planes, i.e., electron channeling, ALCHEMI probes a site-specific elemental concentration from the x-ray spectra or a site-specific chemical state from the electron energy loss spectra, as first demonstrated by Taftø and Krivanek [1].

We revisited and refined the electron channeling EELS [2]. We developed a PC-controlled beam rocking system in combination with the on-line drift correction script working on DigitalMicrograph $^{\mathrm{TM}}$ [3] to perform systematic measurement of a series of ELNES consecutively varying the diffraction conditions with respect to a specific atom plane. Figure 1 shows the PCcontrolled scheme, the degree of deviation being represented by a dimensionless parameter, $w_{\mathrm{g}}=s_{\mathrm{g}} \xi_{\mathrm{g}}$, where $s_{\mathrm{g}}$ and $\xi_{\mathrm{g}}$ are the excitation error and extinction distance of the corresponding beam g. The example spectra of Al- $K$ ELNES of $\mathrm{NiAl}_{2} \mathrm{O}_{4}$ spinel have different weights of two kinds of spectral component of tetrahedrally and octahedrally coordinated Al sites. We resolved their weights and site-specific spectra by applying a multivariate curve resolution (MCR) technique to the raw data [4].

We analyzed the electronic states of transition metal ions at different crystalline sites in $\mathrm{NiFe}_{2} \mathrm{O}_{4}$ and $\mathrm{MnFe}_{2} \mathrm{O}_{4}$ of spinel type crystal structures. Fig.2-a shows the site-specific Fe $L_{2,3}$ ELNES of $\mathrm{NiFe}_{2} \mathrm{O}_{4}$, whose profiles were well reproduced by ab-initio configuration interaction calculations [5]. The calculation details indicated that the low energy shoulder of $L_{3}$ edge was not caused by different valency, but by ligand field splitting.

The transition metal valence states in Mn-containing ferrites have been controversial among several reports. In Fig. 2-b, the experimental site-specific $\mathrm{Mn} L_{2,3}$ spectra are compared with the theoretical. The site-specific spectral difference was explained by mixed valence states of $\mathrm{Mn}^{2+}$ oct and $\mathrm{Mn}^{3+}{ }_{\text {oct }}$.

At the bottom of Figs. 2-a,b, the experimental tetrahedral site intensity fraction at each orientation is compared with simulated one based on the numerical calculations of dynamical diffraction with many beams [6]. The theoretical fraction curves were consistent with the experimental ones, confirming the validity of the present MCR results. The present method will be an important technique especially for analyzing the transition metal oxides in spintronics applications, where transition metal $3 \mathrm{~d}$ electrons at several crystalline sites significantly affect their functionalities.

\section{References}

[1] J. Taftø and O.L. Krivanek, Phys. Rev. Lett. 48 (1982) 560.

[2] K. Tatsumi, S. Muto, I. Nishida and J. Rusz, Appl. Phys. Lett. 96 (2010) 201911.

[3] Y. Sasano and S. Muto, J. Electron Microsc. 57 (2008) 149.

[4] K. Tatsumi and S. Muto, J. Phys.: Condens. Matter 21 (2009) 104213.

[5] H. Ikeno et al., J. Phys.: Condens. Matter 21 (2009) 104208.

[6] J. Rusz, S. Rubino and P. Schattschneider, Phys. Rev. B 75 (2007) 214425. 

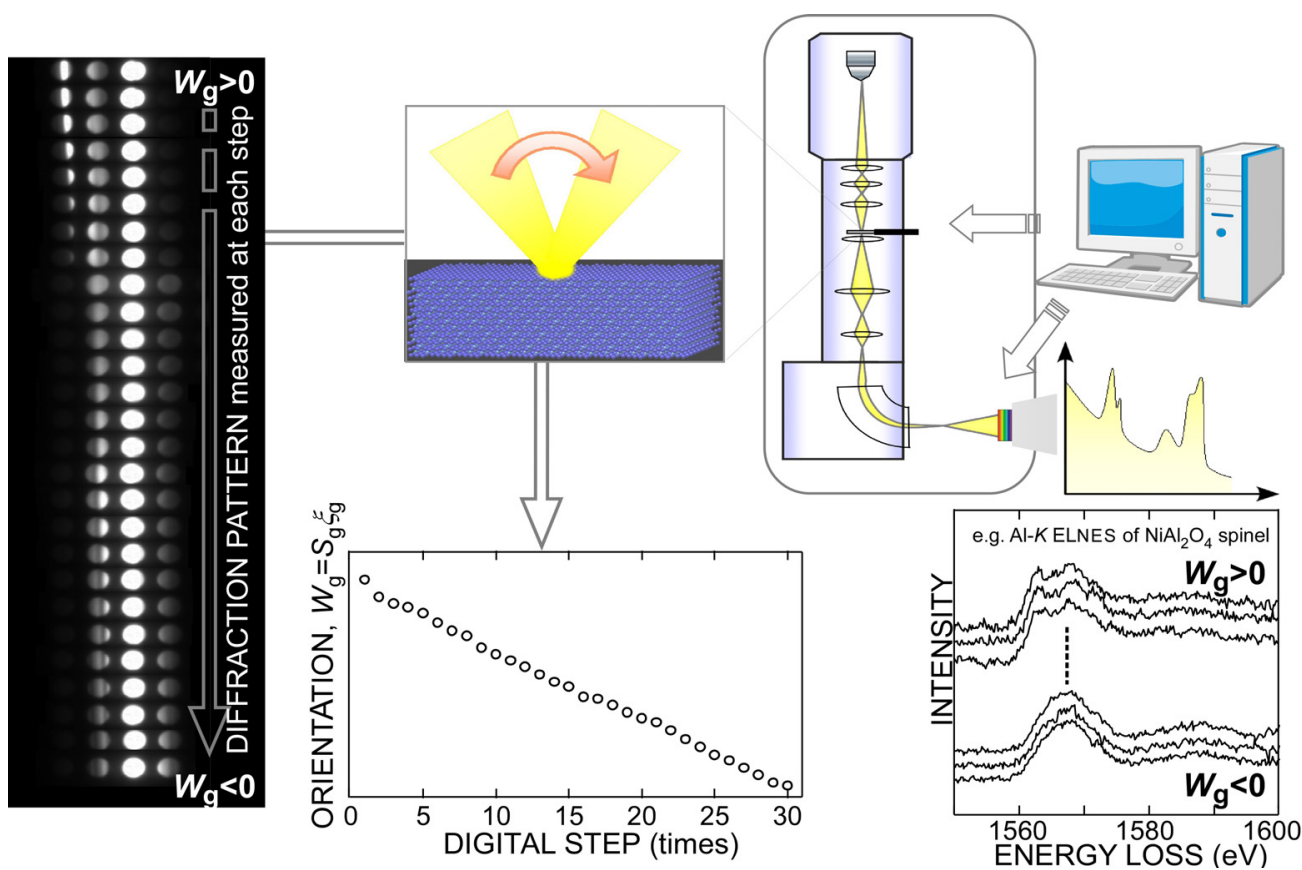

FIG.1 Digital-controlled beam rocking and EELS acquisition system.

a)
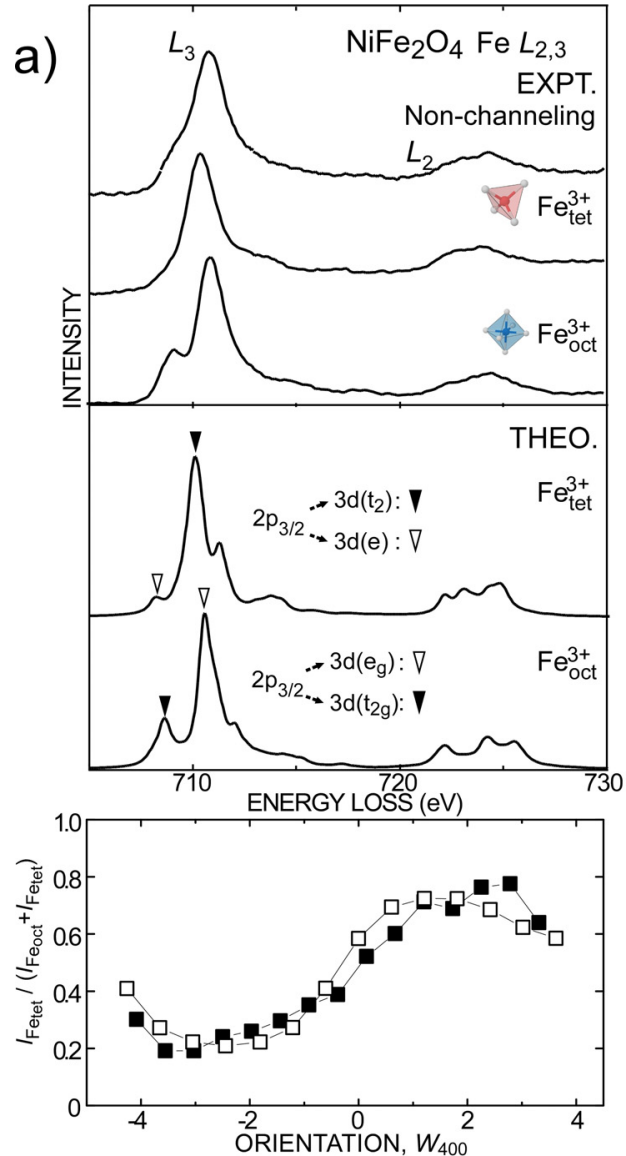

b)
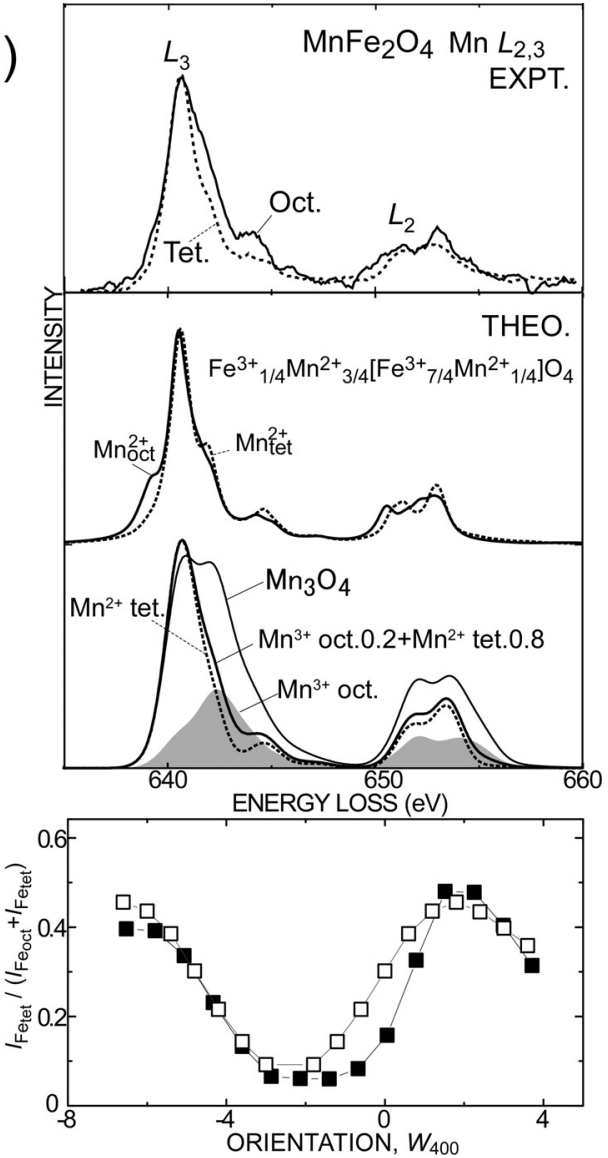

FIG. 2 Site-specific Fe- $L_{2,3}$ ELNES of $\mathrm{NiFe}_{2} \mathrm{O}_{4}$ (a, top) and $\mathrm{Mn}-L_{2,3}$ of $\mathrm{MnFe}_{2} \mathrm{O}_{4}$ (b, top) and fraction of tetrahedral site intensity at each orientation ( $\mathrm{a}$ and $\mathrm{b}$,bottom). 